\title{
Rapamycin protects against aristolochic acid nephropathy in mice by potentiating mammalian target of rapamycin-mediated autophagy
}

\author{
FAN LIN ${ }^{1,2^{*}}$, YUNQI LIU ${ }^{1,3^{*}}$, LILI TANG $^{4}$, XIAOHUI XU ${ }^{2}$, XUELI ZHANG $^{2}$, \\ YIFAN SONG ${ }^{2}$, BICHENG CHEN ${ }^{5}$, YEPING REN ${ }^{2}$ and XIANGDONG YANG ${ }^{1}$ \\ ${ }^{1}$ Department of Nephrology, Qilu Hospital, Shandong University, Jinan, Shandong 250015; \\ ${ }^{2}$ Department of Nephrology, Shenzhen University General Hospital, Shenzhen, Guangdong 518055; \\ ${ }^{3}$ Department of Nephrology, Binzhou Medical University Hospital, Binzhou, Shandong 256600; \\ ${ }^{4}$ Clinical Laboratory, Chinese Medical Hospital of Jining, Jining, Shandong 272037; \\ ${ }^{5}$ Key Laboratory of Diagnosis and Treatment of Severe Hepato-Pancreatic Diseases of Zhejiang Province, \\ The First Affiliated Hospital of Wenzhou Medical University, Wenzhou, Zhejiang 325000, P.R. China
}

Received January 14, 2021; Accepted April 4, 2021

DOI: $10.3892 / \mathrm{mmr} .2021 .12134$

\begin{abstract}
Autophagy serves a crucial role in the etiology of kidney diseases, including drug-induced renal impairment, inherited kidney disease, diabetic nephropathy and aristolochic acid nephropathy (AAN) and is, therefore, a potential target for treatment. We previously demonstrated that rapamycin could attenuate AAN in mice; however, the underlying mechanism remains to be elucidated. Therefore, whether the renal protective effect of rapamycin (an autophagy activator) is related to autophagy in aristolochic acid (AA)-treated mice was of particular interest. The pathophysiological roles of rapamycin were investigated in AA-induced nephrotoxicity in mice and the mechanisms of rapamycin action were explored by evaluating the modulation of autophagy in rapamycin-treated mice and cultured renal tubular epithelial cells. Supplementation with rapamycin reversed AA-induced kidney injury in mice and improved AA-induced autophagy damage in vivo and in vitro. Mechanistically, rapamycin inhibited the renal expression of phosphorylated (p-)mammalian target of rapamycin (mTOR) and p-ribosomal S6 protein kinase 1, which in turn activated renal autophagy and decreased apoptosis, probably by removing AA-elicited damaged mitochondria and misfolded proteins. The findings of the present study demonstrated that rapamycin protects against AA-induced nephropathy
\end{abstract}

Correspondence to: Professor Xiangdong Yang, Department of Nephrology, Qilu Hospital, Shandong University, 107 Wenhuaxi Street, Jinan, Shandong 250015, P.R. China

E-mail: yxd@email.sdu.edu.cn

*Contributed equally

Key words: aristolochic acid nephropathy, mTOR, rapamycin, autophagy, apoptosis by activating the mTOR-autophagy axis and suggested that rapamycin may be a promising pharmacological target for the treatment of AAN.

\section{Introduction}

Aristolochic acid nephropathy (AAN) is a progressive renal tubular interstitial disease caused by the ingestion of Chinese herbal medicines or plants containing aristolochic acid (AA) (1). In Southeast Asia and other countries or regions where traditional Chinese medicine is popular, herbal medicines and plants containing AA are still widely used (2). A multicenter retrospective cohort study in China showed that the incidence of acute renal injury was $11.6 \%$ among nearly 660,000 hospitalized patients, of which $40 \%$ was drug-induced and $16 \%$ may have been caused by Chinese herbal medicine and plants containing AA (3). A considerable proportion of patients with AAN have impaired renal function and progress to end-stage renal disease (ESRD) (4). Patients with ESRD require long-term renal replacement therapy, which necessitates a large number of medical resources. Therefore, an in-depth understanding of the pathogenesis of AAN as well as the development of effective prevention and treatment measures is important.

Rapamycin, a specific inhibitor of mammalian target of rapamycin (mTOR), is a novel and effective immunosuppressant that has a protective effect on the kidney in the state of transplantation $(5,6)$. Rapamycin is reported to improve renal function and renal tissue ultrastructure in a rat renal ischemia-reperfusion injury model by inhibiting renal tubular epithelial cell apoptosis (7). The results of our previous study demonstrated that rapamycin could effectively attenuate AAN (8). However, the mechanism underlying the regulation of AAN by rapamycin remains to be elucidated.

Autophagy is initiated by lysosomes and can be defined as the process by which intracellular fragments, which are widely present in eukaryotic cells, are degraded (9). In this process, 
damaged organelles and misfolded proteins are phagocytized to form autophagosomes, which then fuse with lysosomes to form autolysosomes. These degrade autophagosomes and ensure dynamic cell balance (10). Autophagy is indispensable for maintaining intracellular homeostasis and adapting to stress states (including hunger, energy deprivation, oxidative stress and hypoxia) and ensures cell survival (11). Similarly, AAN leads to organelle dysfunction, including mitochondrial and endoplasmic reticulum stress and induces specific autophagy, resulting in the clearance of damaged organelles (12). However, this is a compensatory mechanism; when stimulation persists or increases, cellular homeostasis is disrupted, leading to apoptosis $(13,14)$. A previous study showed that enhanced renal autophagy can inhibit the apoptosis of kidney cells and serve a protective role in AAN (15). mTOR is a serine-threonine protein kinase that belongs to the phosphatidylinositol 3 kinase protein kinase family (16). It serves a key regulatory role in many biological processes, such as cell proliferation, survival, differentiation and autophagy (17-19). Studies have shown that inhibition of phosphorylated (p-)mTOR can activate autophagy and promote phagocytosis of damaged organelles and misfolded proteins, thereby protecting cells from death $(20,21)$. Nevertheless, as a specific mTOR inhibitor, rapamycin can activate autophagy to maintain AA-stimulated renal cell homeostasis and may, therefore, inhibit apoptosis and exert a protective effect in AAN.

The present study investigated the underlying molecular mechanism of action of rapamycin against AAN. The findings suggested that rapamycin attenuated AAN by blocking the expression of p-mTOR and its downstream substrate p-S6K1, thereby activating autophagy in a mouse model of AAN and in AA-treated renal tubular epithelial cells in vitro, which was associated with the inhibition of cell apoptosis and improved AA-induced renal damage.

\section{Materials and methods}

Animal models. Animal experiments were approved by the Animal Ethics Committee of Shenzhen University (approval no. SUGH-A-02104). A total of 30 six-week-old C57BL/6 male mice weighing 20-25 g were purchased from the Shanghai SLAC Laboratory Animal Co. Ltd. All mice were housed in a room maintained at moderate temperature $\left(20 \pm 2^{\circ} \mathrm{C}\right)$ and humidity (40-60\%) and were subjected to a 12-h light/dark cycle. Mice were provided free access to food and water for the duration of the present study. They were adaptively fed for 7 days and randomly divided into three groups as follows: A control group (Ctrl group, $\mathrm{n}=10$ ), an aristolochic acid-induced group (AAI group, $\mathrm{n}=10$ ) and a rapamycin-treated group (RAP group, $n=10$ ). Mice in the control group were administered $0.9 \%$ normal saline at a volume equivalent to that of the injected drug in the other two groups. The AAI and RAP groups were intraperitoneally injected with aristolochic acid (AA; Sigma-Aldrich; Merck $\mathrm{KGaA}$ ) at a dose of $2.5 \mathrm{mg} / \mathrm{kg} /$ day for 6 weeks. After $2 \mathrm{~h}$, mice in the RAP group were intraperitoneally injected with rapamycin solution (Sigma-Aldrich; Merck KGaA) at a dose of $1 \mathrm{mg} / \mathrm{kg} /$ day. After 6 weeks, all mice were anesthetized with isoflurane (3\% for induction and $2 \%$ for maintenance). Subsequently, $1 \mathrm{ml}$ of arterial blood was quickly drawn from the abdominal aorta. Mice were euthanized by the immediate removal of the heart after exsanguination and death of mice was confirmed by the lack of breathing and reflexes of paw withdrawal. Arterial blood and kidney tissues were collected for subsequent studies.

Serum creatinine and blood urea nitrogen. The supernatant obtained after centrifuging the arterial blood $(300 \mathrm{x} \mathrm{g}$ for $20 \mathrm{~min}$ at $4^{\circ} \mathrm{C}$ ) of each mouse was stored at $-80^{\circ} \mathrm{C}$ until further analysis. Serum creatinine and urea nitrogen levels were detected using enzyme-linked immunosorbent assay kits (Serum creatinine, cat. no. MM-44455M1; serum urea nitrogen, cat. no. MM-0692M1; Jiangsu Baolai Biotechnology Co., Ltd.) according to the manufacturer's protocols.

Hematoxylin and eosin $(H \& E)$ staining. Kidney tissues were fixed in $4 \%(\mathrm{v} / \mathrm{v})$ paraformaldehyde for $48 \mathrm{~h}$ at room temperature, then placed in ethanol solution for gradient dehydration (75, 85, 95 and 100\%) and made transparent in xylene for $30 \mathrm{~min}$. Finally, the transparent kidney tissues were embedded in paraffin and sliced into $5-\mu \mathrm{m}$ thick sections. The slices were incubated overnight in an oven at $65^{\circ} \mathrm{C}$, then dewaxed in xylene for $30 \mathrm{~min}$ and rehydrated in fractionated ethanol series for histological examination. Next, the sections were stained with H\&E (Beijing Solarbio Science \& Technology Co., Ltd.) according to the standardized H\&E staining procedure with Hematoxylin for $10 \mathrm{~min}$ and Eosin for $15 \mathrm{sec}$ at room temperature and observed under a bright field using a DP73 microscope (Olympus Corporation; magnification, x400).

Periodic acid-Schiff (PAS) staining. Sections of renal tissues (5- $\mu \mathrm{m}$ thick) were stained using the PAS staining kit (Muto Pure Chemicals Co., Ltd.) following the manufacturer's instructions. Briefly, sections were treated with $1 \%(w / v)$ periodic acid for $10 \mathrm{~min}$ at room temperature. Then, the sections were treated with Schiff's reagent for $30 \mathrm{~min}$ at $37^{\circ} \mathrm{C}$ after washing three times with distilled water. The staining reaction was terminated using three treatments with sulfurous acid solution. The samples were dried and observed under a bright field using a DP73 microscope (Olympus Corporation; magnification, $\mathrm{x} 400)$.

Cell culture. HK-2 cells were purchased from Kunming Cell Bank, Chinese Academy of Sciences and cultured in DMEM (Gibco; Thermo Fisher Scientific, Inc.) supplemented with $10 \%$ FBS (Gibco; Thermo Fisher Scientific, Inc.) in a $5 \% \mathrm{CO}_{2}$ incubator at $37^{\circ} \mathrm{C}$. HK-2 cells were used for in vitro experiments and were divided into three groups as follows: Untreated group (Control group), HK-2 cells induced with $10 \mu \mathrm{g} / \mathrm{ml} \mathrm{AA}$ for $24 \mathrm{~h}$ (AAI group) and HK-2 cells pre-treated with $50 \mathrm{mM}$ rapamycin for $2 \mathrm{~h}$ and then stimulated with $10 \mu \mathrm{g} / \mathrm{ml}$ AA for $24 \mathrm{~h}$ (RAP group).

Western blotting. RIPA lysis buffer was used to lyse renal tissues and HK-2 cells. After lysis, total proteins were extracted and quantified using a protein assay kit (cat. no. P0006C; Beyotime Institute of Biotechnology). Proteins (40 $\mu \mathrm{g} / \mathrm{lane}$ ) were separated using $10 \%$ sodium dodecyl sulfate-polyacrylamide gels and transferred onto polyvinylidene difluoride membranes, which were blocked with 

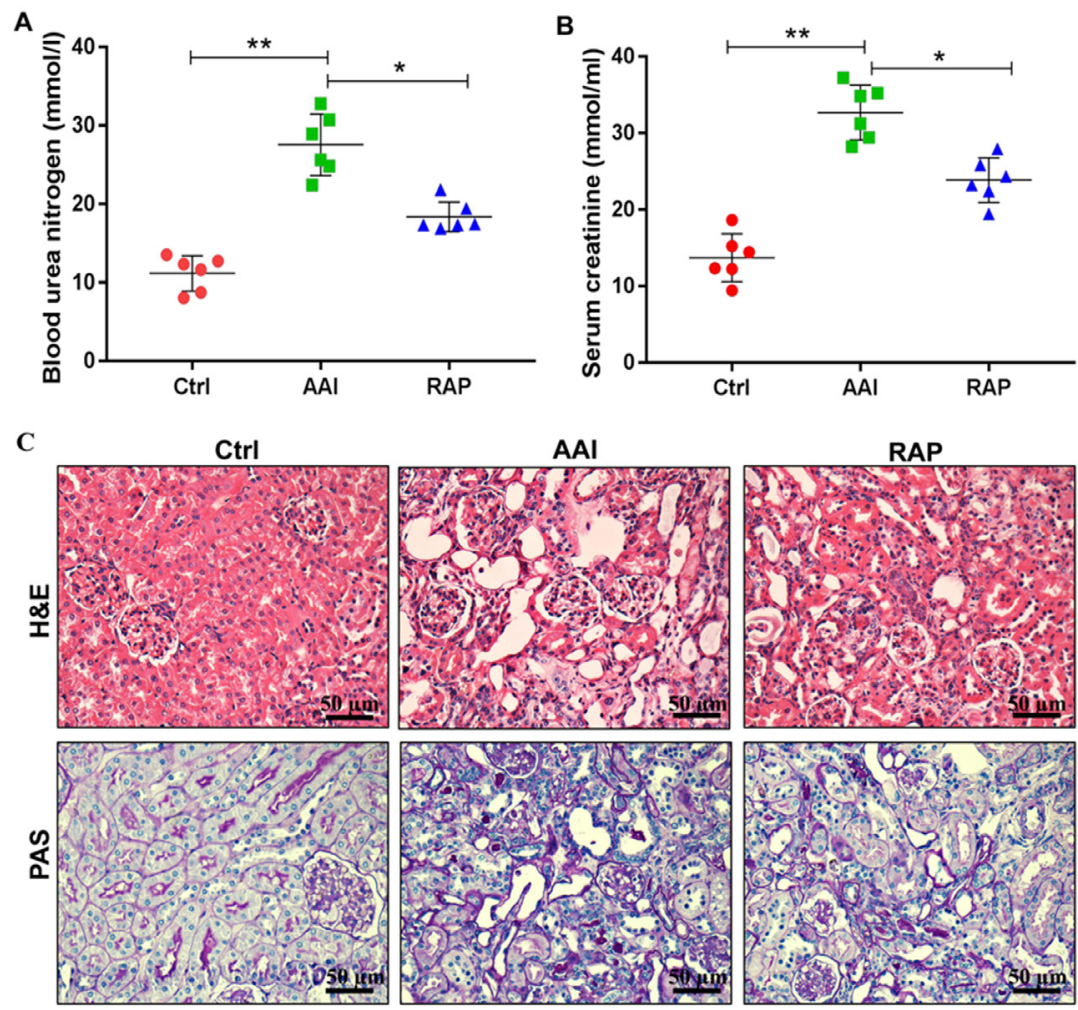

Figure 1. Rapamycin improves AA-induced renal dysfunction and histopathological abnormalities in mice. (A) Levels of blood urea nitrogen and (B) serum creatinine in each group detected using ELISA (n=6/group). (C) Representative microscopic images showing H\&E and PAS staining in the kidney tissues of mice in all groups (scale bar $=50 \mu \mathrm{m} ; \mathrm{n}=6$ /group). Results are presented as the mean \pm standard deviation. ${ }^{*} \mathrm{P}<0.05 ;{ }^{* *} \mathrm{P}<0.01$. All experiments were performed three times. AA, aristolochic acid; H\&E, hematoxylin and eosin staining; PAS, Periodic acid-Schiff staining; Ctrl, control group; AAI, AA-induced group; RAP, rapamycin treatment group.

$5 \%$ fat-free milk for $2 \mathrm{~h}$ at room temperature. The membranes were incubated with primary antibodies against p-mTOR (phospho S2448; cat. no. ab109268; 1:1,000; Abcam), mTOR (cat. no. ab32028; 1:1,000; Abcam), p-ribosomal S6 protein kinase (p-S6K1; phospho T252 for mouse and phospho T229 for human; cat. no. ab59208; 1:1,000; Abcam), S6K1 (cat. no. ab32359; 1:1,000; Abcam), p62 (cat. no. 13121; 1:1,000; Cell Signaling Technology, Inc.), Beclin-1 (cat. no. 3738; 1:1,000; Cell Signaling Technology, Inc.), light chain 3 (LC3; cat. no. 4108; 1:1,000; Cell Signaling Technology, Inc.), Bcl-2 (cat. no. 3498; 1:1,000; Cell Signaling Technology, Inc.) and Bax (cat. no. 2772; 1:1,000; Cell Signaling Technology, Inc.) at $4^{\circ} \mathrm{C}$ overnight and then washed with buffer (TBS with $0.1 \%$ Tween-20). Then, a horseradish peroxidase-conjugated antibody (cat. no. A25012; 1:5,000; Abbkine Scientific Co.) was added to the membranes and samples were incubated at room temperature for $1 \mathrm{~h}$. Lastly, the protein bands were visualized using an enhanced chemiluminescence system. Western primary and secondary antibody removal solution (cat. no. P0025; Beyotime Institute of Biotechnology) was used for re-probing the PVDF membranes. Protein expression levels were normalized to that of GAPDH (cat. no. 5174; $1: 10,000$; Cell Signaling Technology, Inc.) and quantified with ImageJ software (version 1.46; National Institute of Health).

Flow cytometry. HK-2 cells apoptosis in each group were evaluated by flow cytometry using Annexin V-FITC Apoptosis Detection kit (Dojindo Molecular Technologies, Inc.), following the manufacturer's protocol. Briefly, the cells were washed twice with PBS at room temperature, and then re-suspended in $200 \mu \mathrm{l} 1 \mathrm{X}$ AnnexinV Binding Solution and the cell concentration was adjusted to $1 \times 10^{6} / \mathrm{ml}$. Then, $100 \mu \mathrm{l}$ cell suspension was added to a new tube, then Annexin V-FITC (5 $\mu \mathrm{l})$ and PI (5 $\mu \mathrm{l})$ were added and vortexed gently. Following incubation for $15 \mathrm{~min}$ at room temperature in the dark, $400 \mu 1 \mathrm{X}$ Annexin V Binding Solution was added and gently mixed. Finally, the cells were analyzed using a FACScan flow cytometer (BD Biosciences) and FlowJo software (FlowJo LLC 10.0.7) was used to analyze the data. Flow cytometry was performed in triplicate and repeated at least three times.

Immunofluorescent staining. An HK-2 cell monolayer was fixed with 4\% paraformaldehyde at room temperature for $1 \mathrm{~h}$. To detect autophagy, cells were blocked with $10 \%$ normal goat serum with $0.2 \%$ Triton $\mathrm{X}-100$ for $1 \mathrm{~h}$ at room temperature. Cells were then incubated with primary antibodies against LC3B (cat. no. NB100-2220; 1:200; Novus Biologicals, LLC) overnight at $4^{\circ} \mathrm{C}$. After washing three times with PBS, the cells were incubated with Alexa Fluor 488 goat anti-mouse IgG (cat. no. Ab150113; 1:500; Abcam) for $2 \mathrm{~h}$ at room temperature. Subsequently, the cells were washed three times with PBS and stained with 4',6-diamidino-2-phenylindole (Beijing Solarbio Science \& Technology Co., Ltd.) for $30 \mathrm{~min}$ at $37^{\circ} \mathrm{C}$ in the dark. Images were captured using an epifluorescence microscope (Olympus Corporation; magnification, x200). Mean area fraction of LC3B was quantified using Image-Pro plus 6.0 (Media Cybernetics, Inc.). 


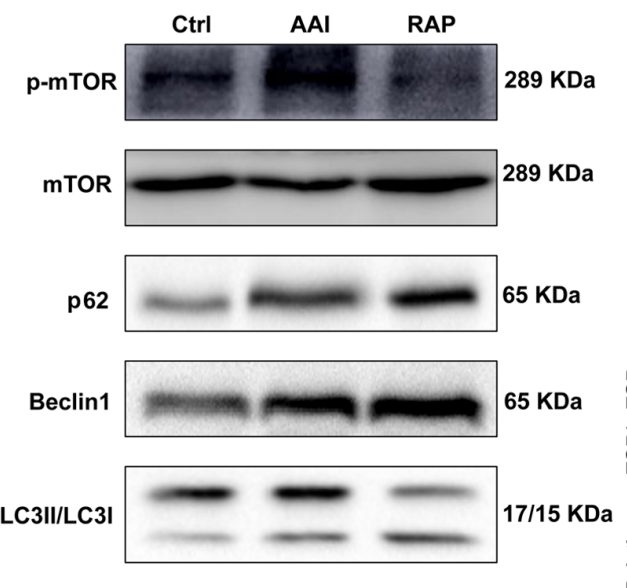

$\mathrm{Bcl}-2$

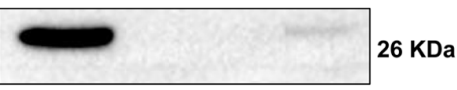

Bax

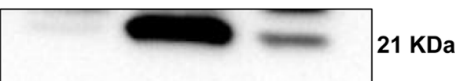

GAPDH

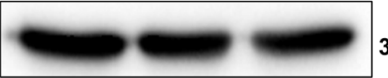

$37 \mathrm{KDa}$

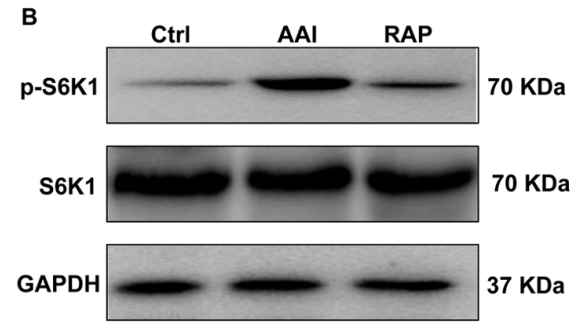

C
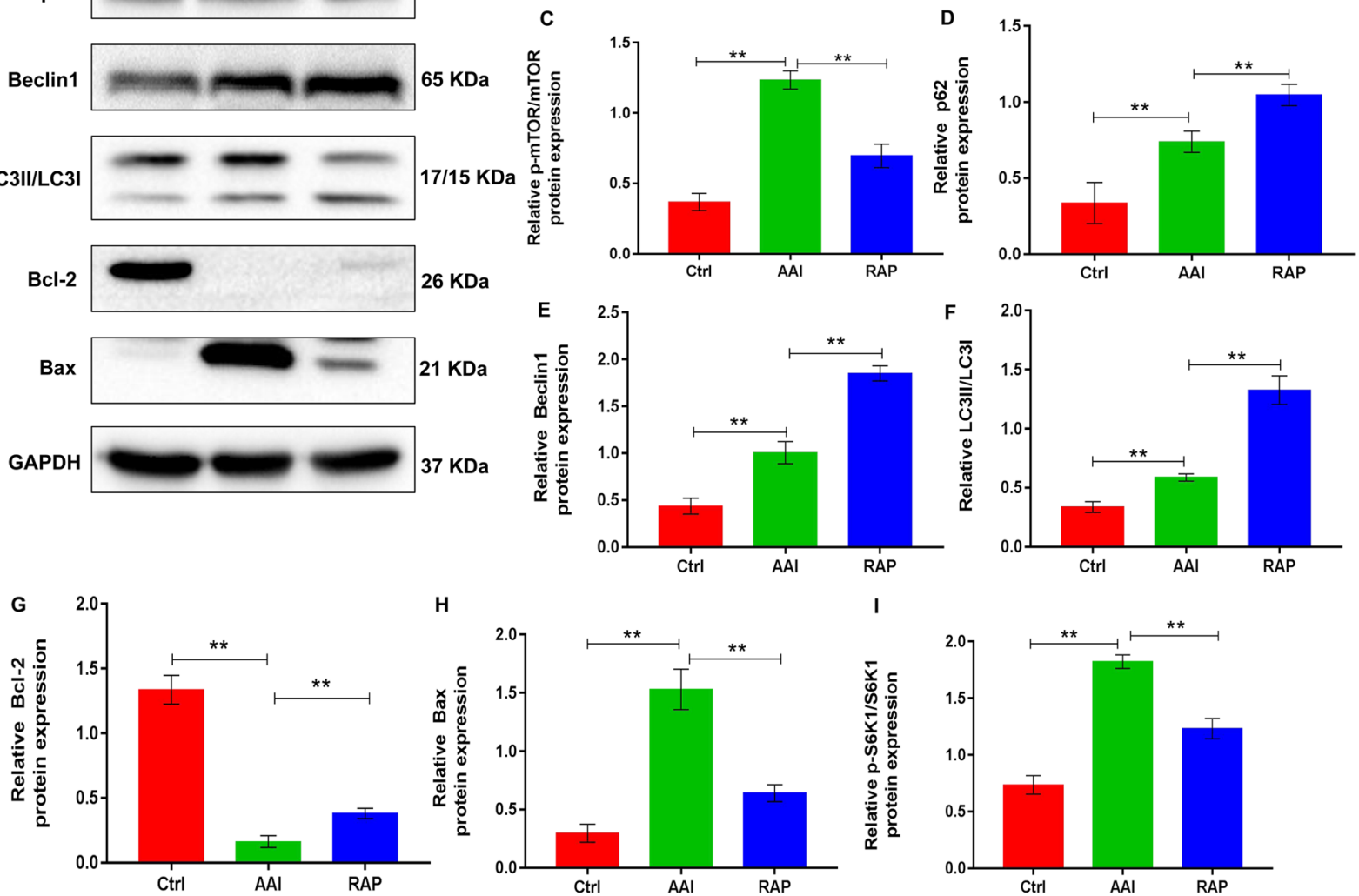

Figure 2. Rapamycin promotes AA-induced renal autophagy and suppresses apoptosis in the kidney tissues of mice by inhibiting mTOR activity. (A) Representative western blots showing the protein expression levels of p-mTOR, mTOR p62, Beclin1, LC3 and Bcl-2 in kidney tissues; GAPDH was used as a loading and normalization control. (B) Representative western blots showing the protein expression levels of p-S6K1 and S6K1 in kidney tissues; GAPDH was used as a loading and normalization control. Renal expression of (C) p-mTOR/mTOR, (D) p62, (E) Beclin1, (F) LC3, (G) Bcl-2, (H) Bax and (I) $\mathrm{p}-\mathrm{S} 6 \mathrm{~K} 1 / \mathrm{S} 6 \mathrm{~K} 1$ were quantified using ImageJ. Results are presented as the mean \pm standard deviation. ${ }^{* *} \mathrm{P}<0.01$. All experiments were performed three times; $\mathrm{n}=10$ /group. AA, aristolochic acid; p-, phosphorylated; LC3, light chain 3; S6K1, ribosomal S6 protein kinase 1; Ctrl, control group; AAI, AA-induced group; RAP, rapamycin treatment group.

Statistical analysis. All experiments were independently repeated three times. Data are presented as the mean \pm standard deviation. GraphPad Prism 8.0 (GraphPad Software, Inc.) was used to analyze the results using one-way analysis of variance. Multiple comparisons between groups were analyzed using Tukey's post-hoc test. $\mathrm{P}<0.05$ was considered to indicate a statistically significant difference.

\section{Results}

Rapamycin ameliorates AA-induced renal dysfunction and structural abnormalities. Studies have shown that AA can lead to renal dysfunction and structural abnormalities in rodents $(22,23)$. Consistent with previous reports, the present study found that AA induced significant renal insufficiency, as evidenced by significantly increased levels of blood urea nitrogen (Fig. 1A) and serum creatinine (Fig. 1B). In addition, H\&E and PAS staining demonstrated that, compared with the
Ctrl group, AAI group mice presented notable tubular dilatation and necrosis, increased tubulointerstitial area, mesangial expansion and glomerular hypertrophy (Fig. 1C). However, these negative effects were reversed following the intraperitoneal injection of rapamycin. Treatment with rapamycin decreased the circulating levels of blood urea nitrogen and serum creatinine and markedly improved renal tubular lesions, mesangial matrix expansion and hypertrophic changes. Collectively, these results indicated that supplementation of rapamycin protects against $\mathrm{AA}$-induced renal damage in mice.

Inhibition of mTOR by rapamycin promotes AA-induced renal autophagy and thereby suppresses apoptosis of kidney tissues in vivo. mTOR serves a significant role in the progression of many diseases and is the main target for regulating autophagy (24). Autophagy is activated in AA-induced renal injury, but further activation of renal autophagy has a protective effect in AAN (15). Autophagy is a method of cellular 

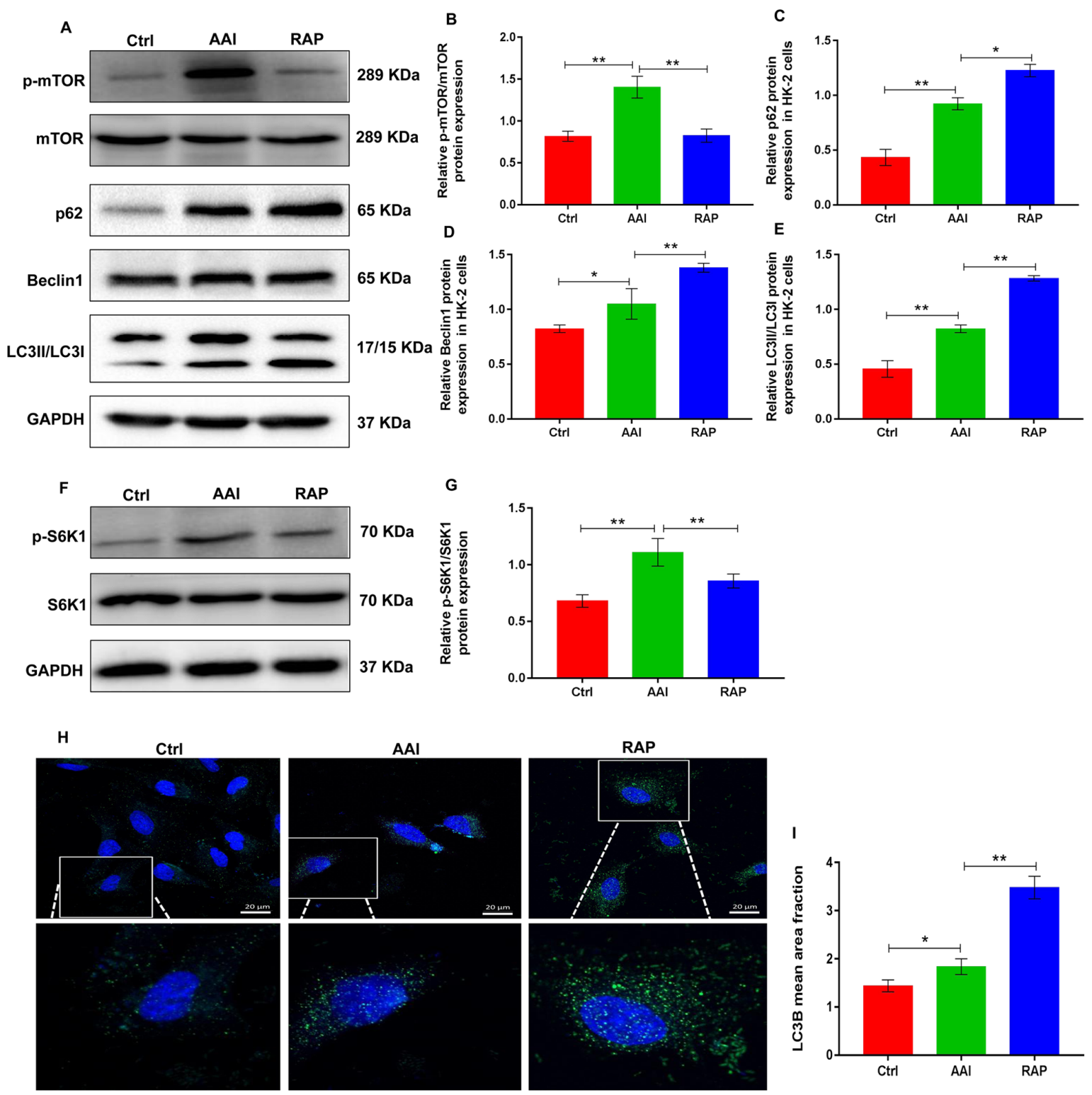

Figure 3. Rapamycin inhibits p-mTOR expression and promotes AA-induced renal autophagy in HK-2 cells. (A) Representative images showing the protein expression of p-mTOR, mTOR, p62, Beclin1 and LC3 in HK-2 cells determined using western blotting. GAPDH was used as an internal control. Relative protein expression of (B) p-mTOR/mTOR, (C) p62, (D) Beclin1 and (E) LC3 were quantified using ImageJ. (F) Representative images showing the protein expression of p-S6K1 and S6K1 in HK-2 cells determined using western blotting. GAPDH was used as an internal control. (G) Relative protein expression of p-S6K1/S6K1 was quantified using ImageJ. (H) Detection of autophagosomes by LC3B immunofluorescence staining in HK-2 cells; small green dots indicate autophagosome formation (scale bar=25 $\mu \mathrm{m}$ ). (I) Mean area fraction of LC3B in each group was quantified using Image-Pro plus 6.0. Results are presented as the mean \pm standard deviation. ${ }^{*} \mathrm{P}<0.05 ;{ }^{* *} \mathrm{P}<0.01$. All experiments were performed three times. p-, phosphorylated; LC3, light chain 3; AA, aristolochic acid; S6K1, ribosomal S6 protein kinase 1; Ctrl, control group; AAI, AA-induced group; RAP, rapamycin treatment group.

adaptation that prevents cell death resulting from external stimulation. During this process, damaged organelles, including the mitochondria and endoplasmic reticulum, are removed $(11,25)$. Therefore, the present study determined whether rapamycin could modulate the renal activity of mTOR in a mouse AAN model, thereby activating renal autophagy and protecting against AA-induced renal damage. To validate the hypothesis, western blotting was performed to determine the renal expression of p-mTOR and its downstream substrate p-S6K1 as well as the autophagic markers, including p62, Beclin1 and LC3II/LCI, in all groups. P-mTOR and p-S6K1 expression was increased in kidney tissues from AA-treated mice with increased renal autophagy, as evidenced by upregulated renal expression of p62, Beclin1 and LC3II/LCI.
As expected, rapamycin treatment effectively prevented the AA-induced increase in mTOR and S6K1 phosphorylation, which led to marked elevations in AA-induced renal expression of the autophagy markers (Fig. 2). This suggested that inhibiting mTOR activity by rapamycin further activated renal autophagy. Autophagy and apoptosis are two connected pathological processes involved in the development of AAN (26). To determine the effects of rapamycin on renal apoptosis in AAN mice, the protein expression of Bcl-2 and Bax, common markers of apoptosis, was assessed in kidney tissues using western blotting. The results indicated that the kidney tissue of AA-treated mice presented with decreased expression of $\mathrm{Bcl}-2$ and increased expression of Bax, which were reversed by rapamycin treatment (Fig. 2A, G and $\mathrm{H}$ ), suggesting that 
A

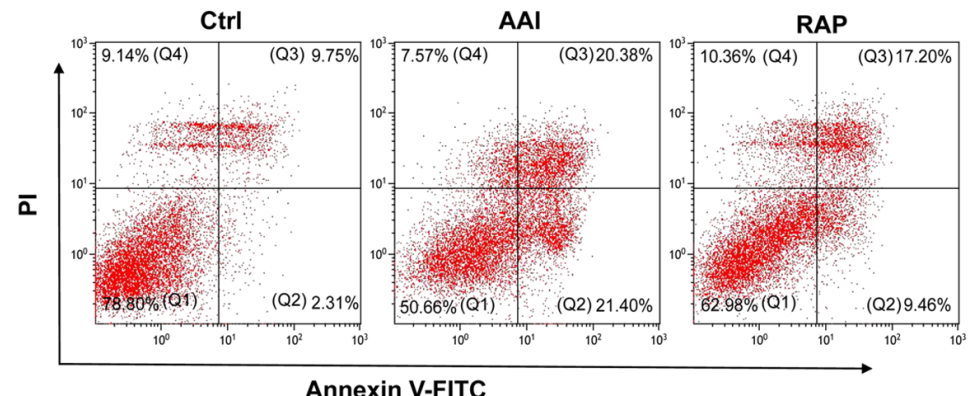

C
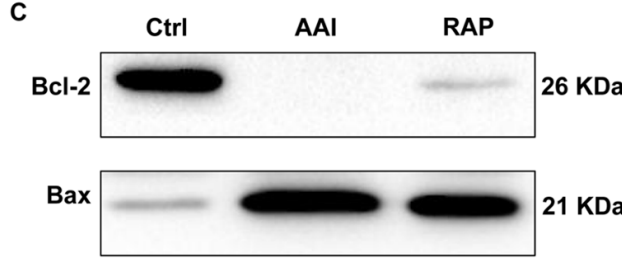

GAPDH

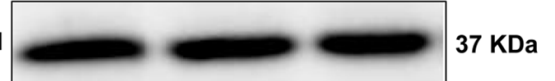

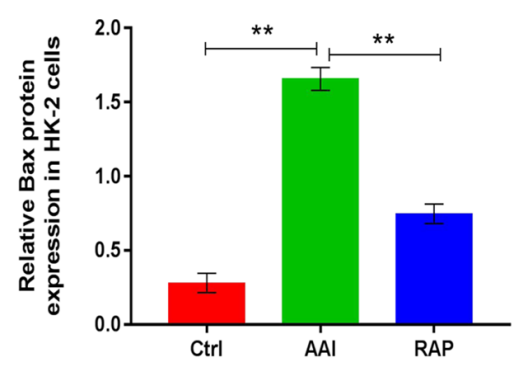

B

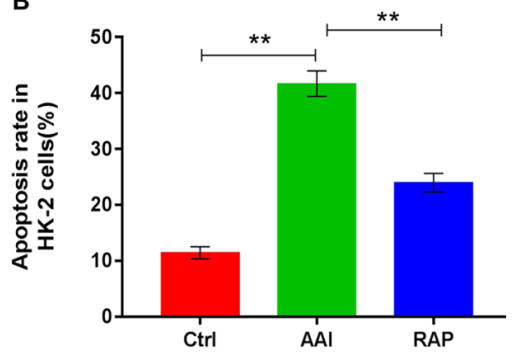

E

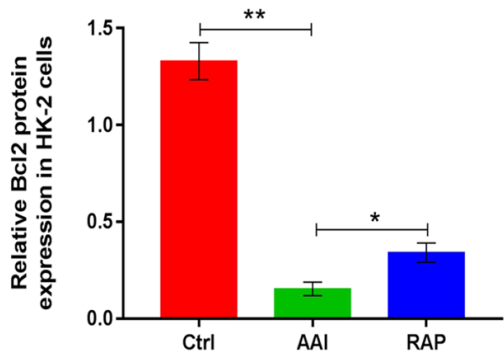

Figure 4. Rapamycin reduces AA-induced apoptosis in HK-2 cells. (A) Flow cytometry results showing apoptosis in HK-2 cells; Q2 for early apoptotic cell death and Q3 for late apoptotic cell death were used to calculate the rates of apoptosis. (B) Cell apoptosis rate (\%) was analyzed using FlowJo software. (C) Representative western blots showing the protein expression of Bcl-2 and Bax in HK-2 cells in each group; GAPDH was used as a loading and normalization control. Protein expression of (D) Bax and (E) Bcl-2 in HK-2 cells was quantified using ImageJ. Results are presented as the mean \pm standard deviation. ${ }^{*} \mathrm{P}<0.05 ;{ }^{* *} \mathrm{P}<0.01$. All experiments were performed three times. AA, aristolochic acid; Ctrl, control group; AAI, AA-induced group; RAP, rapamycin treatment group.

rapamycin inhibited apoptosis in the kidneys of AA-treated renal injury mice. Taken together, these observations indicate that rapamycin supplementation inhibits the renal activity of mTOR, which promotes renal autophagy, thereby probably suppressing the apoptosis of kidney tissues in mice with AA-induced renal injury.

Rapamycin inhibits mTOR activity and promotes AA-induced renal autophagy in $H K-2$ cells. Renal tubular epithelial cells are used to study AAN (27). In the mouse model of the present study, AA treatment induced severe damage to renal tubular epithelial cells. Thus, the pro-autophagy activities of rapamycin was next evaluated using an in vitro experimental model of the AA-challenged HK-2 human renal tubular epithelial cell line. Western blotting revealed that AA treatment markedly increased the expression of p-mTOR and $\mathrm{p}-\mathrm{S} 6 \mathrm{~K} 1$, whereas the treatment of HK-2 cells with rapamycin reduced the AA-induced increase in $\mathrm{p}-\mathrm{mTOR}$ and p-S6K1 (Fig. 3A-B and F-G). Notably, AA treatment resulted in the activation of autophagy in HK-2 cells and was accompanied by increased protein expression of $\mathrm{p} 62$, Beclin-1 and LC3II/LCI. However, the inhibitory action of rapamycin on mTOR further promoted AA-induced autophagy in HK-2 cells, as evidenced by higher protein expression of p62, Beclin-1 and LC3II/LC3I, compared to AA treatment (Fig. 3A-3E). Moreover, cellular immunofluorescence staining with anti-LC3B antibody revealed that rapamycin promoted AA-induced renal autophagy in HK-2 cells (Fig. 3H and I). These data suggested that rapamycin inhibited the activity of mTOR, which mediates stronger autophagy in AA-treated HK-2 cells.
Rapamycin inhibits AA-induced apoptosis in HK-2 cells. The effect of rapamycin on autophagy in AA-treated HK-2 cells may affect cell survival. Therefore, the effects of rapamycin on renal apoptosis was determined using HK-2 cells. Flow cytometry results revealed that AA treatment significantly increased the rate of HK-2 cell apoptosis. Conversely, rapamycin supplementation reversed the AA-induced increase in HK-2 cell apoptosis (Fig. 4A and B). Additionally, to further explore the role of rapamycin in the renal apoptosis of $\mathrm{HK}-2$ cells, the protein expression of $\mathrm{Bcl} 2$ and $\mathrm{Bax}$ was evaluated using western blotting. Following AA treatment, the expression of $\mathrm{Bcl} 2$ was significantly reduced, whereas rapamycin replenishment was associated with a marked elevation in $\mathrm{Bcl} 2$ expression levels compared with that in the AAI group. Treatment with AA notably increased the expression of Bax; however, the AA-induced increase in Bax expression was significantly reduced following treatment with rapamycin compared to that observed in the AAI group (Fig. 4C-4E). Collectively, the observations indicated that rapamycin supplementation inhibited AA-induced apoptosis in HK-2 cells likely by activating autophagy.

\section{Discussion}

The results of the present study provided new insights into the mechanism underlying the improvement of AAN by rapamycin in mice. Rapamycin significantly ameliorated AA-induced renal dysfunction and structural injury. These effects of rapamycin were associated with the promotion of renal autophagy and inhibition of kidney-cell apoptosis. The data demonstrated, for the first time to the best of the 
authors' knowledge, that rapamycin protects the kidney from AA-induced renal damage by potentiating the mTOR-mediated autophagy signaling pathway.

AAN, a variant of interstitial nephritis leading to ESRD and urothelial malignancy, was originally reported in Belgium in a group of patients who ingested slimming pills made from powdered root extracts of Chinese herbs containing AA (28). Unfortunately, there is no proven and effective treatment method for AAN. The present study demonstrated that blocking the mTOR signaling pathway by rapamycin effectively ameliorated AAN, as evidenced by a reduction in blood urea nitrogen and serum creatinine levels, as well as an improvement in the structural units of the kidney tissues. The findings suggested that rapamycin may be a promising pharmacological strategy for the treatment of AAN. However, the renal protective mechanism of rapamycin in $\mathrm{AAN}$ requires further investigation.

Autophagy is an adaptive response to intracellular or extracellular stress, including hypoxia, nutrient or growth factor deprivation, oxidative damage and other destructive injuries (29). Stress-induced autophagy supports cell survival by clearing and recovering damaged macromolecules, protein aggregates and organelles (30). Thus, it is generally regarded as a self-protection mechanism in cells. However, upon persistent stimulation or beyond a certain threshold, this protective effect is compromised and eventually leads to cell death (31). Accumulating evidence indicates that autophagy is predominantly regulated by the mTOR-dependent signaling pathway (32). As a specific mTOR inhibitor, rapamycin binds to mTOR complex I through fk506 binding protein 12 and directly inhibits the protein expression of p-mTOR (33). Notably, previous studies have demonstrated that renal autophagy is activated in AAN; furthermore, enhanced renal autophagy can prevent AA-induced renal damage in mice $(14,15)$. Thus, it was hypothesized that the nephroprotective role of rapamycin in AAN is probably mediated by mTOR-induced activation of autophagy. Consistent with the findings of the abovementioned studies, the present study showed that AA treatment for 6 weeks resulted in a sharp increase in p-mTOR and its downstream substrate p-S6K1 expression and a significant elevation in p62, Beclin-1 and LC3II/LC3I expression in the kidney tissues of mice. By contrast, replenishment of rapamycin reversed the increased expression of p-mTOR and p-S6K1 and induced the protein expression of p62, Beclin-1 and LC3II/LC3I. Consistent with these results, the direct treatment of HK-2 cells with AA, with or without rapamycin supplementation, resulted in similar findings. The results suggested that rapamycin inhibited the activity of mTOR, which in turn, mediated stronger autophagic activity in AAN. The activation of renal autophagy induced by AA may be a compensatory stress-protective mechanism; continuous AA stimulation decompensates this adaptive protection and does not inhibit the progression of AAN. Rapamycin significantly inhibited the phosphorylation of mTOR and S6K1, which resulted in further activation of renal autophagy and exerted a cytoprotective effect to maintain the survival of renal cells that were under AA stimulation. These findings explained the renal protective mechanism of rapamycin in AAN. Notably, AA induced high expression of p-mTOR and also activated autophagy in mouse kidney tissues and HK-2 cells. This may be due to mTOR exerting other biological effects in AAN, which are independent of AA-induced renal autophagy. Other physiological and pathological effects of mTOR, including the regulation of cell differentiation, inflammatory action and glucose and lipid metabolism in AAN, are worthy of further research.

Autophagy and apoptosis are two important cellular processes that involve complex and intersecting protein networks (34). Autophagy promotes cell survival by accelerating cellular metabolism and assisting the removal of mitochondria following cellular injury, which can regulate apoptosis (35). Mitochondrial damage induces the release of metabolic hydrolase and caspase activator into the cytoplasm from the mitochondria, which degrades certain proteins in the cytoplasm and induces apoptosis (36). However, activated autophagy intercepts and degrades damaged mitochondria and also selectively removes activated caspase- 8 to prevent apoptosis $(37,38)$. The present study demonstrated that AA treatment promotes apoptosis in HK-2 cells in vitro and the renal tissues of mice in vivo. Although the inhibition of mTOR phosphorylation by rapamycin further activated renal autophagy in a mouse model of AAN and AA-challenged HK-2 cells, it is unclear whether the induction of autophagy by rapamycin is responsible for apoptosis observed in the present study. Rapamycin markedly inhibited apoptosis in AA-treated HK-2 cells and renal tissues of mice. Taken together, the data supported a role for rapamycin as an effector to promote AA-induced autophagy, which probably thereby inhibited apoptosis in AAN by decreasing the renal expression of p-mTOR.

The present study had some limitations. First, although other studies have demonstrated that enhanced renal autophagy can inhibit the apoptosis of kidney cells and serve a protective role in AAN $(12,15)$, whether the beneficial effect of mTOR inhibition on apoptosis disappears in the case of co-treatment with an autophagy inhibitor, such as the chloroquine, should be investigated. In the future, further experiments will be conducted to verify this hypothesis. Second, the inhibitory effect of rapamycin on mTOR activity in untreated with AA cells should be verified to ensure the rationality of the experimental grouping, which is also a limitation that should be noted for future experiment group design.

To summarize, the present study demonstrated that rapamycin was highly effective in preventing the progression of AAN. Functionally, it was found that rapamycin effectively inhibited the renal activity of mTOR signal pathway in AAN, which in turn, promoted renal autophagy, thus probably enhancing the anti-apoptosis ability of kidney cells and protecting mice from AA-induced renal injury. Therefore, because rapamycin has been used clinically as an effective immunosuppressant to prevent renal transplant rejection, it shows potential as a therapy for future medical interventions in the management of AAN.

\section{Acknowledgements}

Not applicable.

\section{Funding}

The present study was supported by grants from the National Natural Science Foundation of China of Xiangdong Yang 
(grant no. 81670660); the Shandong Important Research Plans Fund of Xiangdong Yang (grant no. GG201809250293); the Natural Science Foundation of Shenzhen University General Hospital of Fan Lin (grant no. SUGH2018QD071); and the Natural Science Foundation of Shenzhen University General Hospital of Yeping Ren (grant no. SUGH2020QD011).

\section{Availability of data and materials}

All data used or analyzed during the present study are available from the corresponding author on reasonable request.

\section{Authors' contributions}

FL, YQL, LLT, XHX and YPR performed the experiments. XDY and BCC conceived and designed the study. FL, YFS and XLZ analyzed the data and drafted the manuscript. XDY and YQL reviewed and edited the manuscript. XDY and FL assessed and confirmed the authenticity of all the raw data. All authors read and approved the final version of the manuscript.

\section{Ethics approval and consent to participate}

Animal experiments were approved by the Animal Ethics Committee of Shenzhen University (approval no. SUGH-A-02104).

\section{Patient consent for publication}

Not applicable.

\section{Competing interests}

The authors declare that they have no competing interests.

\section{References}

1. Jadot I, Declèves AE, Nortier J and Caron N: An Integrated View of Aristolochic Acid Nephropathy: Update of the Literature. Int J Mol Sci 18: 18, 2017.

2. Luciano RL and Perazella MA: Aristolochic acid nephropathy: Epidemiology, clinical presentation, and treatment. Drug Saf 38: 55-64, 2015.

3. Xu X, Nie S, Liu Z, Chen C, Xu G, Zha Y, Qian J, Liu B, Han S, $\mathrm{Xu} \mathrm{A}$, et al: Epidemiology and Clinical Correlates of AKI in Chinese Hospitalized Adults. Clin J Am Soc Nephrol 10: 1510-1518, 2015.

4. Redeker S, Oppe M, Visser M, Busschbach JJV, Weimar W, Massey E and Ismail S; 'Nierteam aan Huis' consortium: Cost-effectiveness of a home-based group educational programme on renal replacement therapies: A study protocol. BMJ Open 9: e025684, 2019.

5. Hahn D, Hodson EM, Hamiwka LA, Lee VW, Chapman JR, Craig JC and Webster AC: Target of rapamycin inhibitors (TOR-I; sirolimus and everolimus) for primary immunosuppression in kidney transplant recipients. Cochrane Database Syst Rev 12: CD004290, 2019.

6. Tarasewicz A, Dębska-Ślizień A, Rutkowska B, Szurowska E and Matuszewski M: Efficacy and safety of mammalian target of rapamycin inhibitor use-long-term follow-up of first tuberous sclerosis complex patient treated de novo with sirolimus after kidney transplantation: a case report. Transplant Proc 50: 1904-1909, 2018.

7. Yang X, Yan X, Yang D, Zhou J, Song J and Yang D: Rapamycin attenuates mitochondrial injury and renal tubular cell apoptosis in experimental contrast-induced acute kidney injury in rats. Biosci Rep 38: 38, 2018.
8. Wu M, Tang L, Chen B, Zheng J, Dong F, Su Z and Lin F: Blockade of the mTOR signaling pathway with rapamycin ameliorates aristolochic acid nephropathy. Exp Ther Med 19: 2887-2894, 2020

9. Yu L, Chen Y and Tooze SA: Autophagy pathway: Cellular and molecular mechanisms. Autophagy 14: 207-215, 2018.

10. Mizushima N and Komatsu M: Autophagy: Renovation of cells and tissues. Cell 147: 728-741, 2011.

11. Levine B and Kroemer G: Biological Functions of Autophagy Genes: A Disease Perspective. Cell 176: 11-42, 2019.

12. Zeng Y, Li S, Wu J, Chen W, Sun H, Peng W, Yu X and Yang X: Autophagy inhibitors promoted aristolochic acid I induced renal tubular epithelial cell apoptosis via mitochondrial pathway but alleviated nonapoptotic cell death in mouse acute aritolochic acid nephropathy model. Apoptosis 19: 1215-1224, 2014.

13. Yang CC, Wu CT, Chen LP, Hung KY, Liu SH and Chiang CK: Autophagy induction promotes aristolochic acid-I-induced renal injury in vivo and in vitro. Toxicology 312: 63-73, 2013.

14. Jin C, Miao X, Zhong Y, Han J, Liu Q, Zhu J, Xia X and Peng $X$ : The renoprotective effect of diosgenin on aristolochic acid I-induced renal injury in rats: Impact on apoptosis, mitochondrial dynamics and autophagy. Food Funct 11: 7456-7467, 2020.

15. Zeng Y, Yang X, Wang J, Fan J, Kong Q and Yu X: Aristolochic acid I induced autophagy extenuates cell apoptosis via ERK 1/2 pathway in renal tubular epithelial cells. PLoS One 7: e30312, 2012.

16. Wu Y, Li W, Hu Y, Liu Y and Sun X: Suppression of sirtuin 1 alleviates airway inflammation through mTOR mediated autophagy. Mol Med Rep 22: 2219-2226, 2020.

17. Wang S, Livingston MJ, Su Y and Dong Z: Reciprocal regulation of cilia and autophagy via the MTOR and proteasome pathways. Autophagy 11: 607-616, 2015.

18. Li H, Zhang Y, Liu S, Li F, Wang B, Wang J, Cao L, Xia T, Yao Q, Chen $\mathrm{H}$, et al: Melatonin enhances proliferation and modulates differentiation of neural stem cells via autophagy in hyperglycemia. Stem Cells 37: 504-515, 2019.

19. He J, Ma J, Ren B and Liu A: Advances in systemic lupus erythematosus pathogenesis via mTOR signaling pathway. Semin Arthritis Rheum 50: 314-320, 2020.

20. Shi B, Ma M,Zheng Y, Pan Y and Lin X: mTOR and Beclin1: Two key autophagy-related molecules and their roles in myocardial ischemia/reperfusion injury. J Cell Physiol 234: 12562-12568, 2019.

21. Kim YC and Guan KL: mTOR: A pharmacologic target for autophagy regulation. J Clin Invest 125: 25-32, 2015.

22. Kim JY, Leem J and Jeon EJ: Protective effects of melatonin against aristolochic acid-induced nephropathy in mice. Biomolecules 10: 11, 2019.

23. Zhang HM, Zhao XH, Sun ZH, Li GC, Liu GC, Sun LR, Hou JQ and Zhou W: Recognition of the toxicity of aristolochic acid. J Clin Pharm Ther 44: 157-162, 2019.

24. Munson MJ and Ganley IG: MTOR, PIK3C3, and autophagy: Signaling the beginning from the end. Autophagy 11: 2375-2376, 2015.

25. Wang NN, Dong J, Zhang L, Ouyang D, Cheng Y, Chen AF, Lu AP and Cao DS: HAMdb: A database of human autophagy modulators with specific pathway and disease information. J Cheminform 10: 34, 2018

26. Luo Z, Xu X, Sho T, Zhang J, Xu W, Yao J and Xu J: ROS-induced autophagy regulates porcine trophectoderm cell apoptosis, proliferation, and differentiation. Am J Physiol Cell Physiol 316: C198-C209, 2019.

27. Huang X, Wu J, Liu X, Wu H, Fan J and Yang X: The protective role of Nrf2 against aristolochic acid-induced renal tubular epithelial cell injury. Toxicol Mech Methods 30: 580-589, 2020.

28. Debelle FD, Vanherweghem JL and Nortier JL: Aristolochic acid nephropathy: A worldwide problem. Kidney Int 74: 158-169, 2008.

29. Kim KH and Lee MS: Autophagy - a key player in cellular and body metabolism. Nat Rev Endocrinol 10: 322-337, 2014.

30. Filomeni G, De Zio D and Cecconi F: Oxidative stress and autophagy: The clash between damage and metabolic needs. Cell Death Differ 22: 377-388, 2015.

31. Mizushima $\mathrm{N}$ and Levine B: Autophagy in human diseases. N Engl J Med 383: 1564-1576, 2020.

32. Papadopoli D, Boulay K, Kazak L, Pollak M, Mallette F, Topisirovic I and Hulea L: mTOR as a central regulator of lifespan and aging. F1000 Res 8: F1000 Faculty Rev-998, 2019. 
33. Sato M, Seki T, Konno A, Hirai H, Kurauchi Y, Hisatsune A and Katsuki H: Rapamycin activates mammalian microautophagy. J Pharmacol Sci 140: 201-204, 2019.

34. Maiuri MC, Zalckvar E, Kimchi A and Kroemer G: Self-eating and self-killing: Crosstalk between autophagy and apoptosis. Nat Rev Mol Cell Biol 8: 741-752, 2007.

35. Onishi M, Yamano K, Sato M, Matsuda N and Okamoto K: Molecular mechanisms and physiological functions of mitophagy. EMBO J 40: e104705, 2021.

36. Abate M, Festa A, Falco M, Lombardi A, Luce A, Grimaldi A, Zappavigna S, Sperlongano $\mathrm{P}$, Irace $\mathrm{C}$, Caraglia $\mathrm{M}$, et al: Mitochondria as playmakers of apoptosis, autophagy and senescence. Semin Cell Dev Biol 98: 139-153, 2020.
37. Wang K: Autophagy and apoptosis in liver injury. Cell Cycle 14: 1631-1642, 2015.

38. D'Arcy MS: Cell death: A review of the major forms of apoptosis, necrosis and autophagy. Cell Biol Int 43: 582-592, 2019.

(i) (3) This work is licensed under a Creative Commons

cc. $\mathrm{EY}$ No ND Attribution-NonCommercial-NoDerivatives 4.0 International (CC BY-NC-ND 4.0) License. 